\title{
Intracellular Acidosis Promotes Mitochondrial Apoptosis Pathway: Role of EMMPRIN Down-regulation via Specific Single-chain Fv Intrabody
}

\author{
Patcharin Thammasit ${ }^{1}$, Sirikwan Sangboonruang ${ }^{1}$, Supattara Suwanpairoj², Wannisa Khamaikawin ${ }^{2}$, \\ Nutjeera Intasai ${ }^{3}$, Watchara Kasinrerk ${ }^{2,4}$, Chatchai Tayapiwatana ${ }^{2,4,5}$, and Khajornsak Tragoolpua ${ }^{\boxplus}$ \\ 1. Division of Clinical Microbiology, Department of Medical Technology, Faculty of Associated Medical Sciences, Chiang Mai University, Chiang \\ Mai, Thailand \\ 2. Division of Clinical Immunology, Department of Medical Technology, Faculty of Associated Medical Sciences, Chiang Mai University, Chiang \\ Mai, Thailand \\ 3. Division of Clinical Microscopy, Department of Medical Technology, Faculty of Associated Medical Sciences, Chiang Mai University, Chiang Mai, \\ Thailand \\ 4. Biomedical Technology Research Unit, National Center for Genetic Engineering and Biotechnology, National Science and Technology Develop- \\ ment Agency at the Faculty of Associated Medical Sciences, Chiang Mai University, Chiang Mai, Thailand \\ 5. BioMedical Engineering Center, Chiang Mai University, Chiang Mai, Thailand
}

$\square$ Corresponding author: Khajornsak Tragoolpua, Ph.D., Division of Clinical Microbiology, Department of Medical Technology, Faculty of Associated Medical Sciences, Chiang Mai University, Chiang Mai 50200, Thailand. Telephone: (66) 5394-5086 ext. 18; Fax: (66) 5394-6042; e-mail: khajornsak.tr@cmu.ac.th

() 2015 Ivyspring International Publisher. Reproduction is permitted for personal, noncommercial use, provided that the article is in whole, unmodified, and properly cited. See http://ivyspring.com/terms for terms and conditions.

Received: 2014.10.22; Accepted: 2014.12.31; Published: 2015.01.20

\begin{abstract}
Extracellular matrix metalloproteinase inducer (EMMPRIN) is a human leukocyte surface molecule that is enriched on the surface of many cancer cells, and it plays an important role in proliferation and metastasis. In this study, we utilized the chimeric adenoviral vector Ad5/F35 carrying gene encoding scFv against EMMPRIN (scFv-M6-IB9) to down-regulate EMMPRIN cell surface expression and investigated programmed cell death response in colorectal cancer (CRC) cell, Caco-2. The scFv-M6-1B9 intrabody exhibits robust activity in reducing EMMPRIN cell surface expression. This approach led to the inducing of apoptosis, which was relative to the increasing of apoptotic bodies in sub-Gl peak, phosphatidylserine externalization, as well as TUNEL-positive cells. In addition, real-time RT-PCR and western blotting analysis indicated that apoptosis was enhanced through the mitochondrial pathway, a marked reduction of $\mathrm{Bcl}-2$, leading to the translocation of cytochrome $c$ and also the dramatic activation of caspase-3. Moreover, carcinoembryonic antigen (CEA), a tumor marker for CRC, was found to have significantly diminished in both secreted protein and mRNA levels. In conclusion, these findings suggest that EMMPRIN down-regulation by scFv-M6-1B9 intrabody has great potential in enhancing the efficacy of apoptosis induction through the mitochondrial pathway and in effecting a decline in the CEA level. Thus, its benefits could be applied to project the future prospects for targeted gene therapy and therapeutic application in monitoring colorectal cancer.
\end{abstract}

Key words: EMMPRIN, scFv-M6-1B9 intrabody, apoptosis, colorectal cancer cells, carcinoembryonic antigen (CEA)

\section{Introduction}

Based on colorectal cancer therapy regimen is the surgical removal of solid tumor masses, usually combined with the chemical treatments. However, chemotherapy reaches low efficiency levels with the emergence of resistant tumors and severe side effects $[1,2]$. For this reason, several researches in recent years have been looking to shift the approach as regards designing an alternative treatment. Strategies 
for targeted therapy have gained immense attention in comparison with the conventional treatment [3]. The understanding of apoptosis has enabled the formation of a basis for novel targeted therapeutic approaches capable of targeting signaling pathways that are essential for inducing death in cancer cells [4]. This has been shown to be a novel therapeutic approach of inducing apoptosis of cancer cells and should be helpful in developing more effective treatments for colorectal cancer.

Apoptosis has been speculated as the major mechanism for cancer treatment, particularly in chemotherapy-induced cell death, besides being the focus of many preclinical drug investigations [5]. Likewise, immunotherapy for cancer requires an apoptosis-sensitive phenotype of target cells, for example, in the case of cetuximab-induced apoptosis through the blocking of the epidermal growth factor receptor (EGFR) [6]. Many studies have revealed that extracellular matrix metalloproteinase inducer (EMMPRIN) related with cancer cell apoptosis mediates in a hyaluronan-dependent manner via the PI3K and Erk1/2 pathways [7-11]. EMMPRIN, or CD147, is a highly glycosylated cell surface transmembrane glycoprotein that belongs to the Ig superfamily [12, 13]. Overexpression of this molecule has been noted on the surface of many cancer cells, and it is found to promote multiple properties including cell proliferation, invasiveness, metastasis, angiogenesis, glycolysis, and multi-drug resistance (MDR) [10, 14]. In 2006, Yang et al. reported that the knock-down of EMMPRIN/CD147 expression by RNA interference (RNAi) sensitized cancer cells to anoikis, as demonstrated by caspase-3 activation, increased DNA fragmentation, and decreased cellular viability in human breast cancer cells [15]. Compared to other similar technologies aimed at the ablation of protein function, such as intracellular antibody or intrabody, this technology knockout is at the post-translational level. It has more specific subcellular compartments due to the addition of localization signal sequences as well as precise epitopes on the target proteins [16].

Several studies have demonstrated the potential of intrabodies in therapeutic applications. The first promising intrabody approaches in cancer treatment which have been published in immunotherapy involve the application of single-chain variable fragment $(\mathrm{scFv})$ against human epidermal growth factor receptor 2 (HER2) in breast cancer [17]. Until now, $\mathrm{scFv}$ intrabody has been proven to be promising in several types of cancer [18-22]. Recently, our group generated the functional intrabody against EMMPRIN (scFv-M6-1B9) and transferred the gene encoding scFv-M6-1B9 intrabody via adenovirus serotype 5 vector (Ad5-scFv-M6-1B9) which retained
EMMPRIN in ER with the KDEL peptide (ER retention signal) [23].

Gene transfer systems are basically classified into non-viral and viral transfer systems. At present, the main disadvantage of non-viral vector is the low transduction efficiency and transient transgene expression, no specific cell targeting and difficult in vivo applications [24]. The retroviral vectors are suitable for ex vivo gene therapy, and despite the disadvantages, retroviral gene delivery systems have been used already in a number of clinical trials [24, 25]. However, integration of retrovirus genomes into the host genome might induce mutagenesis in some clinical applications [26]. Adenoviruses (Ad) have been adopted as potential delivery vehicles in vector system for cancer gene therapy [27]. They rapidly infect a broad range of human cells as well as tend to yield high levels of gene transfer compared to the levels achieved with other currently available vectors. Rein et al. achieved higher clinical efficacy of adenoviral vector-mediated strategies in cervical cancer gene therapy [28]. Furthermore, Ad vectors bearing chimeric fiber (Ad5/F35), Ad5 fiber tail domain and Ad35 fiber shaft and knob, were developed and found to have high transduction efficiencies [29]. Since CD46 is a receptor for Ad5/F35 which is highly expressed in most cancer cells, chimeric adenovirus 5/F35 (Ad5/F35) was observed to have high potency for transduction in various human cancer cell lines [30].

In our study, we demonstrated earlier that chimeric adenovirus 5/F35 carrying gene encoding scFv against EMMPRIN (Ad5/F35-scFv-M6-1B9) is effective in diminishing EMMPRIN cell surface expression and subsequently decreasing cancer cell proliferation, invasion, and metastasis in the colorectal cancer cell line, Caco-2 [31]. However, the apoptotic effect of reducing EMMPRIN cell surface levels using intrabody technique in established colorectal cancer cells has hitherto been unclear. Therefore, the aim of the present study was to investigate the molecular mechanism by which EMMPRIN cell surface down-regulation via scFv-M6-1B9 intrabody induces apoptosis in the colorectal cancer cell line, Caco-2. Moreover, carcinoembryonic antigen (CEA) monitoring to predict the response toward down-regulating the EMMPRIN molecule was investigated. These findings might provide a new strategy that would lead to the development of targeted-therapy and its role in the management of colorectal cancer.

\section{Materials and Methods}

\section{Cell culture}

Human colorectal cancer cell line Caco-2 (kindly 
obtained from Assoc. Prof. Dr. Samlee Mankhetkorn, Laboratory of Physical Chemistry, Molecular and Cellular Biology, Department of Radiologic Technology, Faculty of Associated Medical Sciences, Chiang Mai University, Chiang Mai, Thailand) was cultured in Dulbecco's Modified Eagle's medium (DMEM) (Invitrogen, Green Island, NY) supplemented with $10 \%$ fetal bovine serum (FBS), penicillin (100 Units $/ \mathrm{mL})$, and streptomycin $(100 \mu \mathrm{g} / \mathrm{mL})$, and incubated in a humidified atmosphere with $5 \% \mathrm{CO}_{2}$ at $37^{\circ} \mathrm{C}$.

\section{Chimeric adenoviruses}

The chimeric adenovirus 5/F35 harboring scFv-M6-1B9 gene which encoding intrabody specific to EMMPRIN (Ad5/F35-scFv-M6-1B9) and the adenovirus harboring $\mathrm{scFv}$ irrelevant intrabody (Ad5/F35-scFv-irrelevant), both carrying the green fluorescent protein (GFP) reporter gene, were generated as described in another research review [31].

\section{EMMPRIN expression}

The EMMPRIN expression of the Caco-2 cell was analyzed using western blotting analysis. In brief, untransduced, Ad5/F35-scFv-irrelevant, or Ad5/F35$s c F v-M 6-1 B 9$ transduced cell lysates were prepared in the compartments of the membrane and cytosol by using FractionPREPTM Cell Fractionation Kit (BioVision, Milpitas, CA) by following the instructions of the manufacturer and supplemented with protease inhibitor cocktail set I (Calbiochem, Darmstadt, Germany). The protein concentration of each fraction was quantified using a BCA protein assay kit (Merck, Darmstadt, Germany). Equal amounts of protein samples $(40 \mu \mathrm{g})$ were separated on $15 \%$ sodium dodecyl sulphate (SDS)-polyacrylamide gels and transblotted onto polyvinylidene difluoride (PVDF) membranes (Merck, Darmstadt, Germany). For immunoblotting detection, the membranes were incubated overnight with $1 \mu \mathrm{g} / \mathrm{mL}$ of rabbit anti-EMMPRIN pAb (Invitrogen, Burlington, Canada). The antibodies were diluted in a blocking buffer composed of $5 \%$ skim milk in phosphate buffered saline (PBS). Blots were subsequently incubated for 1 $h$ with peroxidase-conjugated goat anti-rabbit-IgG pAb (KPL, Madison, WI). After the washing step, the reactions were visualized using luminata forte western HRP substrate (Millipore, Billerica, MA) and exposed to Hyperfilm ${ }^{\mathrm{TM}}$ ECL (Thermo Scientific, Rockford, IL). The band density was measured using the ImageQuant TL software (GE Healthcare, Piscataway, $\mathrm{NJ})$.

\section{Apoptosis induction}

Caco- 2 cells $\left(1 \times 10^{5} /\right.$ well $)$ transduced with 100 MOI of Ad5/F35-scFv-irrelevant or Ad5/F35-scFv-M6-
1B9. After $48 \mathrm{~h}$ of transduction, the cells were harvested and washed twice with cold PBS. The supernatant was discarded and the cells were resuspended with $300 \mu \mathrm{L}$ of cold PBS, followed by fixation of the cells with $700 \mu \mathrm{L}$ absolute ethanol and incubation on ice for $30 \mathrm{~min}$. Then, the cells were washed twice and $1 \mathrm{~mL}$ of propidium iodide (PI) solution $(20 \mu \mathrm{g} / \mathrm{mL}$ PI, 0.1\% TritonX-100, $2 \mathrm{mM}$ EDTA, and $8 \mu \mathrm{g} / \mathrm{mL}$ RNase) was added. Finally, the cells were analyzed using FACSort flow cytometer and cell quest software (Becton Dickinson, Franklin Lakes, NJ).

\section{Phosphatidylserine externalization}

The externalization of phosphatidylserine was measured using a FACSort flow cytometer by following the instructions provided with the Annexin V-PE apoptosis detection kit (Calbiochem, Darmstadt, Germany). In brief, after transducing the Caco-2 cells with Ad5/F35-scFv-irrelevant or Ad5/F35-scFv-M6$1 B 9$ at MOI of 100 for $48 \mathrm{~h}$, the cells were harvested, washed twice with cold PBS, and resuspended in 500 $\mu \mathrm{L}$ of binding buffer. Then, the cells were stained with $5 \mu \mathrm{L}$ of PE-conjugated Annexin V/PI and incubated at room temperature for $5 \mathrm{~min}$ in the dark. Finally, the cells were analyzed by flow cytometry.

\section{DNA fragmentation}

DNA fragmentation was carried out using the In Situ Cell Death Detection Kit, TMR red (Roche, Mannheim, Germany), according to the instructions provided by the manufacturer. Briefly, after $48 \mathrm{~h}$ of transduction with $100 \mathrm{MOI}$ of Ad5/F35-scFv-irrelevant or Ad5/F35-scFv-M6-1B9, the cells were fixed with the fixative solution (4\% Paraformaldehyde in PBS $\mathrm{pH}$ 7.4) for $1 \mathrm{~h}$ at room temperature. The cells were washed twice and incubated with the permeabilization solution $(0.1 \%$ Triton $\mathrm{X}-100$ in $0.1 \%$ Sodium citrate) for $2 \mathrm{~min}$ on ice. After the permeabilization, the cells were washed twice and incubated with $50 \mu \mathrm{L}$ of TUNEL reaction mixture (label solution and enzyme solution) for $1 \mathrm{~h}$, at $37^{\circ} \mathrm{C}$, in the dark. The cells were then washed twice and analyzed by confocal laser scanning microscopy (CLSM) (Nikon Instruments, Melville, NY) and flow cytometry.

\section{Expression of mRNAs of apoptosis-related genes}

The effects of EMMPRIN down-regulation via scFv-M6-1B9 intrabody on apoptosis-related gene expression were determined by quantitative real-time RT-PCR assay. The total RNA from Caco-2 cells transduced for $48 \mathrm{~h}$ was extracted using Total RNA Isolation NucleoSpin ${ }^{\circledR}$ RNA II (Clontech, Mountain View, CA), according to the instructions provided by the manufacturer. Complementary DNA (cDNA) 
were obtained by reverse transcription (RT) of $0.7 \mu \mathrm{g}$ total RNA, using oligo $(\mathrm{dT})_{18}$ primers in the RevertAid $^{\mathrm{TM}}$ First Strand cDNA Synthesis (Thermo Scientific, Rockford, IL) by following the enzyme supplier's instructions.

Real-time RT-PCR assays for the quantitative determination of the mRNAs were performed using the MiniOpticon ${ }^{\mathrm{TM}}$ Real-Time PCR Detection System (Bio-Rad, Hercules, CA). The amplifications were performed in $25 \mu \mathrm{L}$ mixtures containing $2.5 \mu \mathrm{L}$ cDNA preparation and $2 \times \mathrm{SYBR}$ Green qPCR Master Mix (Thermo Scientific, Rockford, IL), according to the instructions provided by the manufacturer. The primer sets for Bcl-2, cytochrome $c$, and caspase- 3 are listed in Table 1 . The PCR reactions were amplified with initial denaturation at $95^{\circ} \mathrm{C}$ for $10 \mathrm{~min}$, followed by 40 cycles of denaturation at $95^{\circ} \mathrm{C}$ for $15 \mathrm{sec}$, annealing at $56^{\circ} \mathrm{C}$ for $30 \mathrm{sec}$, and extension at $60^{\circ} \mathrm{C}$ for 1 $\mathrm{min}$. After the final cycle, the reaction was held in the final extension step at $60^{\circ} \mathrm{C}$ for $5 \mathrm{~min}$. Melting curve analysis was performed to ensure that the specific desired amplicon was detected. Glyceraldehyde-3-phosphate dehydrogenase (GAPDH) was used as the internal control. The normalized mRNA expression was analyzed using the comparative $C_{T}$ method [32].

\section{Expression of apoptosis-related proteins}

The effects of EMMPRIN down-regulation via scFv-M6-1B9 intrabody on the expression of apoptosis-related proteins were determined using western blotting analysis. Untransduced, Ad5/F35-scFvirrelevant, or Ad5/F35-scFv-M6-1B9 transduced cell lysates were prepared using RIPA buffer supplemented with a mixture of protease inhibitors (Merck, Darmstadt, Germany). To determine the translocation of cytochrome $c$ from mitochondria to the cytosol, the compartments were separated using the Mitochondria/Cytosol Fractionation Kit (BioVision, Milpitas, CA), according to the instructions provided by the manufacturer. Immunodetection was carried out with rabbit anti-Bcl-2 mAb (1:1000) [mouse anti-cytochrome $c$ mAb (1:500) or mouse anti-caspase-3 $\mathrm{mAb}(1: 500)]$ in 5\% skim milk in PBS (all the antibodies were purchased from Merck, Darmstadt, Germany). This step was followed by incubation with peroxidase-conjugated goat anti-rabbit-IgG pAb (KPL, Madison, WI) or anti-mouse-IgG pAb (Merck, Darmstadt, Germany).The reactions were visualized using luminata forte western HRP substrate and the mixture was immediately exposed to Hyperfilm ${ }^{\mathrm{TM}}$ ECL.

\section{Carcinoembryonic antigen (CEA) level}

\section{CEA secretion}

The CEA secretion level was examined using the automated ADVIA Centaur ${ }^{\circledR}$ CEA assay system (Siemens Diagnostics, Deerfield, IL) based on the two-site sandwich immunoassay which uses direct chemiluminometric technology. The Caco- 2 cells $\left(1 \times 10^{4}\right.$ per well) were plated on a 24-well tissue culture plate and transduced with $100 \mathrm{MOI}$ of Ad5/F35-scFv-irrelevant or Ad5/F35-scFv-M6-1B9 at $5 \% \mathrm{CO}_{2}$, at $37^{\circ} \mathrm{C}$. The cultured medium was changed at a regular time interval of 2 days with half volume. After 7 days and 10 days of incubation, the supernatant was collected and the CEA concentration was evaluated using the automated analyzer [33].

\section{CEA mRNA expression}

The effect of EMMPRIN down-regulation on the CEA mRNA level was also determined by quantitative real-time RT-PCR assay, as described previously. RT-PCR was performed using the CEA primer set [34] and listed in Table 1. GAPDH was performed as the internal control. The normalized mRNA expression was determined using the comparative $\mathrm{C}_{\mathrm{T}}$ method.

Table 1. List and sequence of primers

\begin{tabular}{lll}
\hline Primers & Sequences & $\begin{array}{l}\text { Refer- } \\
\text { ences }\end{array}$ \\
\hline Bcl-2_Fwd & 5-TCGCCCTGTGGATGACTG-3 & {$[66]$} \\
Bcl-2_Rev & 5-CAGGAGAAATCAAACAGAGGC-3 & \\
Cytochrome c_Fwd & 5-GAGCGGGAGTGTTCGTTGT-3 & {$[66]$} \\
Cytochrome c_Rev & 5-GTCTGCCCTTTCTTCCTTCT-3 & \\
Caspase-3_Fwd & 5'-TCACAGCAAAAGGAGCAGTTT-3' & {$[67]$} \\
Caspase-3_Rev & 5'-CGTCAAAGGAAAAGGACTCAA-3' & \\
CEA_Fwd & 5'-CCATGGAGTCTCCCTCG-3' & {$[34]$} \\
CEA_Rev & 5'-GTAGCTTGCTGTGTCATTTC-3' & \\
GAPDH_Fwd & 5'-GAAGGTGAAGGTCGGAGTC-3' & [68] \\
GAPDH_Rev & 5'-GAAGATGGTGATGGGATTTC-3' & \\
\hline
\end{tabular}

\section{Statistical analysis}

The results were presented as the mean \pm s.e.m. of the three independent experiments. The comparison between the groups was carried out by one-way ANOVA using the SPSS Statistics 17.0 software and statistical significance was set at 95\% $(p<0.05)$.

\section{Results}

\section{Accumulation of EMMPRIN inside cell medi- ated scFv-M6-1B9 intrabody}

Our previous study revealed for the first time that scFv-M6-1B9 intrabody has the ability to down-regulate EMMPRIN cell surface expression on colorectal cancer cell, Caco-2, via flow cytometry [31]. To confirm the reduction of the EMMPRIN cell surface expression due to its accumulation inside the cells, immunoblotting was performed. The reduction of EMMPRIN cell surface expression had been demonstrated in the membrane fraction protein of the 
Caco-2 cells expressing scFv-M6-1B9 intrabody by $50 \%$, compared to untransduced and irrelevant control. Interestingly, increasing accumulation of EMMPRIN was found in the cytoplasmic fraction protein of the Caco-2 cells expressing scFv-M6-1B9 intrabody, as shown in Figure 1. This indicates that the scFv-M6-1B9 intrabody dramatically reduced EMMPRIN cell surface expression and that these molecules accumulated inside the Caco-2 cells.

\section{Induction of Caco-2 cell apoptosis by EMMPRIN down-regulation mediated scFv-M6-1B9 intrabody}

To determine whether EMMPRIN down-regulation via scFv-M6-1B9 intrabody could influence apoptosis, apoptotic induction in Caco-2 cells transduced with Ad5/F35-scFv-irrelevant and Ad5/F35-scFv-M6-1B9 was analyzed using flow cytometry. Transduced GFP-positive cells were gated for further analysis of PI staining. Apoptotic cells with DNA content being lessthan the G1-phase cells, coupled with the reduced DNA content of the apoptotic nuclei, resulted in the hypodiploid DNA peak observed in the DNA fluorescence histogram. The percentage of apoptosis in the Caco-2 cells expressing scFv-M6-1B9 intrabody (43.6\%) was observed to be significantly higher than the percentage in the scFv-irrelevant control $(4.2 \%)$, as shown in Figure 2A.

To confirm whether phosphatidylserine exter- nalization had any effect during the development of apoptosis, Annexin V assay was performed. After $48 \mathrm{~h}$ of incubation, transduced GFP positive cells were gated and further analyzed to determine the percentage of PE conjugated Annexin V/PI-positive cells. Caco-2 cells expressing scFv-M6-1B9 intrabody were observed to have increased Annexin $\mathrm{V}$-positive cells (39.1\%) (Annexin V+/PI- and Annexin V+/PI+) compared with the irrelevant control $(7.0 \%)$ as presented in Figure 2B.

TUNEL assay was performed for detecting DNA fragmentation, a characteristic hallmark of apoptosis, by confocal laser scanning microscopy (CLSM) and flow cytometry. DNA fragmentation was observed to be superior in Caco-2 cells expressing scFv-M6-1B9 intrabody than in irrelevant $\mathrm{scFv}$ intrabody (Figure $2 \mathrm{C})$. To quantify the DNA fragmentation in the Caco-2 cells, transduced GFP positive cells were gated and the TUNEL-positive cells were further analyzed using flow cytometry. As illustrated in Figure 2D, the percentage of TUNEL-positive cells in the Caco-2 cells expressing scFv-M6-1B9 intrabody was observed to undergo a significant increase of $13.19 \%$. As with TUNEL staining, there were no differences between the cells expressing irrelevant $\mathrm{scFv}$ intrabody and the untransduced cells. The result demonstrates that Caco-2 cells expressing scFv-M6-1B9 intrabody exhibit an increment in DNA fragmentation compared to irrelevant control.
A

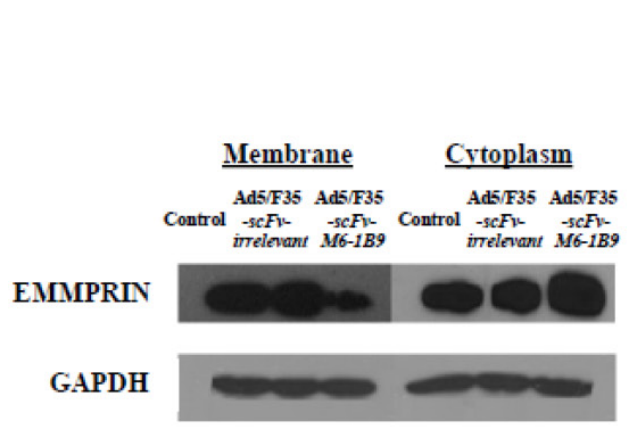

B

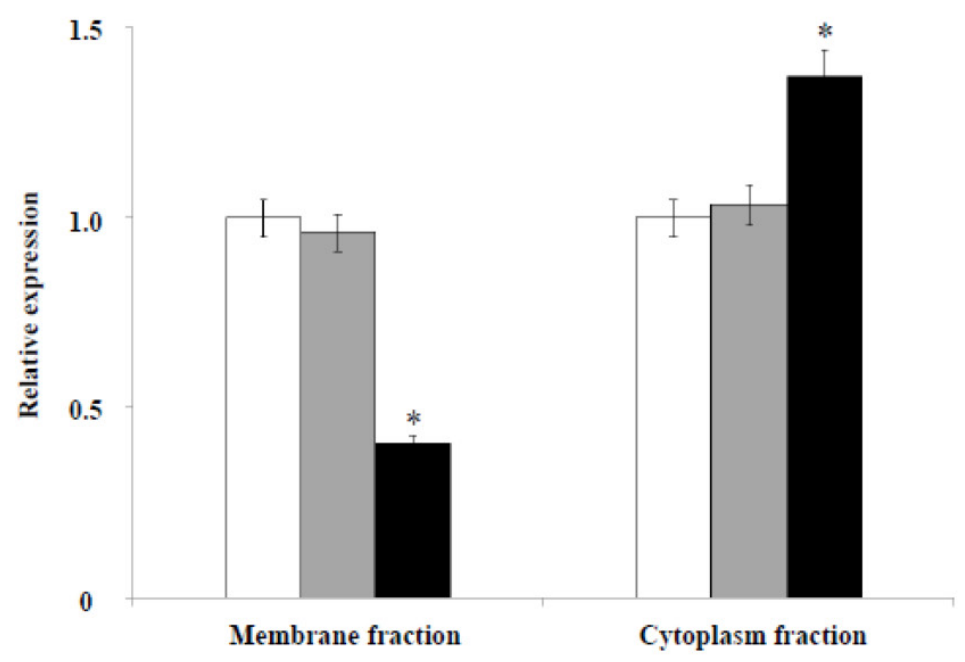

Figure 1. Down-regulation of EMMPRIN cell surface expression via scFv-M6-1B9 intrabody and its accumulation inside Caco-2 cells. (A) Western blotting analysis of EMMPRIN protein expression and (B) densitometry analysis of EMMPRIN expression. The white, gray, and black bars represent the protein fractions from untransduced, Ad5/F35-scFv-irrelevant, and Ad5/F35-scFv-M6-IB9 transduced Caco-2 cells, respectively. Y-axis is the fold change of the EMMPRIN expression which was normalized with GAPDH. $* p<0.05$, as determined by one-way ANOVA. 
A

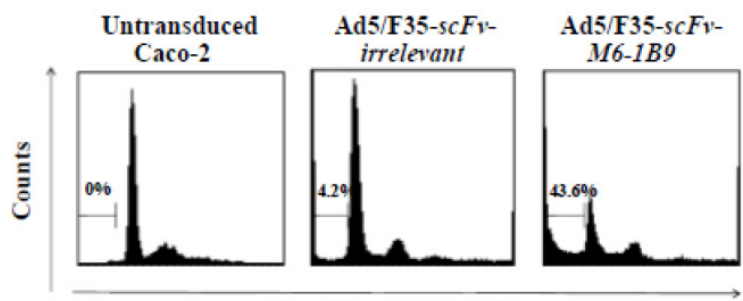

Propidium iodide (PI)
B

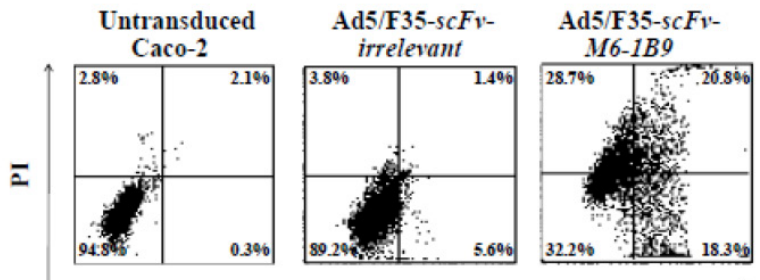

AnnexinV-PE
C

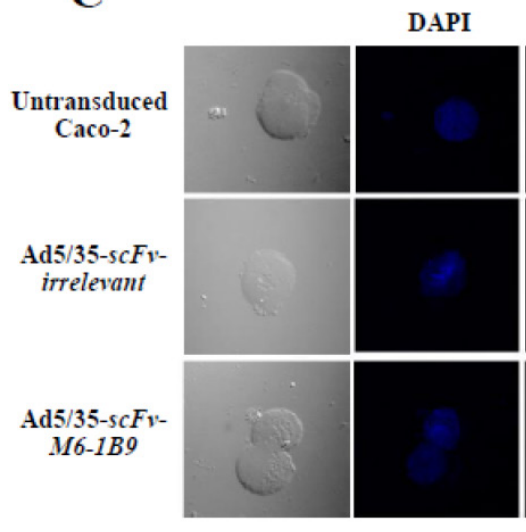

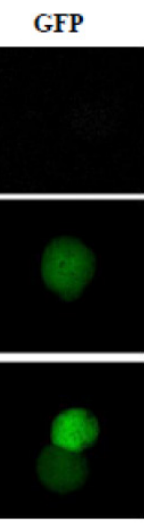

TUNEL

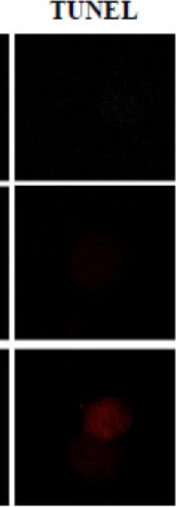

D

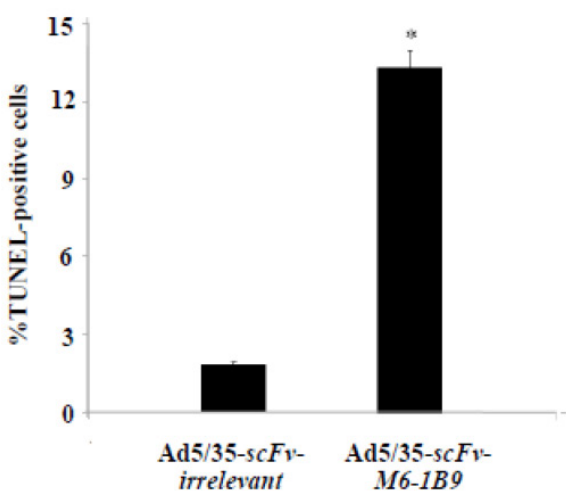

Figure 2. EMMPRIN down-regulation induced apoptosis in Caco-2 cells. Caco-2 cells were transduced with Ad5/F35-scFv-irrelevant or Ad5/F35-scFv-M6-IB9. Transduced GFP positive cells were used for the apoptosis studies. (A) FACS analysis with PI staining for the detection of apoptotic cells. The histogram presents the percentage of apoptotic cells in sub-Gl peak, which was calculated as the percentage of all the transduced cells. (B) Detection of phosphatidylserine externalization by Annexin V assay. The dual parametric dot plot shows the viable cell population in the bottom left quadrant (Annexin V- PI-), the early apoptotic cells in the bottom right quadrant (Annexin $\mathrm{V}^{+} \mathrm{Pl}-$ ), the late apoptotic cells in the top right quadrant (Annexin $\left.\mathrm{V}^{+} \mathrm{PI}^{+}\right)$, and the necrotic cells in the top left quadrant (Annexin $\mathrm{V}-\mathrm{PI}^{+}$). Identification of DNA fragmentation by TUNEL assay, using (C) CLSM and (D) FACS analysis. Representative fluorescent images of TUNEL showed DAPI (blue) stained nuclei, transduced cells (green), and TUNEL-positive cells (red). * $p<0.05$, as determined by one-way ANOVA.

\section{Activation of mitochondrial apoptosis signaling in Caco-2 cells expressing scFv-M6-1B9 intra- body}

To further examine the possible apoptosis mechanism involved in the EMMPRIN down-regulation of Caco-2 cells, quantitative real-time RT-PCR was utilized to analyze the mRNA expressions of Bcl-2, cytochrome $\mathrm{c}$, and caspase- 3 . The results revealed that caspase- 3 mRNA expression had markedly increased in the Caco-2 cells expressing scFv-M6-1B9 intrabody compared with the irrelevant control cells $(p<0.05)$, as shown in Figure 3A. We found no appreciable alterations in the $B c l-2$ and $c y$ tochrome $c$ mRNA expression levels in the Caco- 2 cells expressing scFv-M6-1B9 intrabody and the irrelevant control.

We then investigated the effect of this protein expression mediated EMMPRIN down-regulation in Caco-2 cells via scFv-M6-1B9 intrabody. The western immunoblotting technique was used to detect the expression of Bcl-2, cytochrome $c$, and caspase- 3 . The expression of GAPDH was used as the internal control to reveal equal loading of protein samples. Our data showed that the down-regulation of EMMPRIN reduced the expression of the $\mathrm{Bcl}-2$ protein and the translocation of cytochrome $c$ from mitochondria into cytosol. Moreover, the cleaved caspase-3 protein was most remarkable in the Caco-2 cells expressing scFv-M6-1B9 intrabody. Contrarily, all the proteins were not found altered in untransduced and irrelevant control cells. Thus, these results indicate that EMMPRIN down-regulation by scFv-M6-1B9 intrabody has the ability to activate Caco- 2 cells apoptosis via mitochondrial pathway.

\section{Monitoring of CEA expression level}

The objective was to examine the effect of EMMPRIN down-regulation by scFv-M6-1B9 intrabody on CEA release from Caco-2 cells. CEA secretion was examined by measuring the CEA concentration in the Caco-2 cell culture supernatant. Noteworthily, more than $50 \%$ inhibition of the CEA release was produced in the Caco-2 cells expressing scFv-M6-1B9 intrabody after 10 days of incubation, as demonstrated in Figure 4A. Contrary to this observation, the untransduced and irrelevant control cells both showed high CEA levels in the supernatant. 
To further determine the effect of EMMPRIN down-regulation by scFv-M6-1B9 intrabody on CEA mRNA expression in Caco-2 cells, real-time RT-PCR was performed. The results revealed the CEA mRNA expression after 7 days and 10 days of incubation of the Caco-2 cells. Significant reduction in the CEA mRNA expression was detected in the Caco-2 cells

A

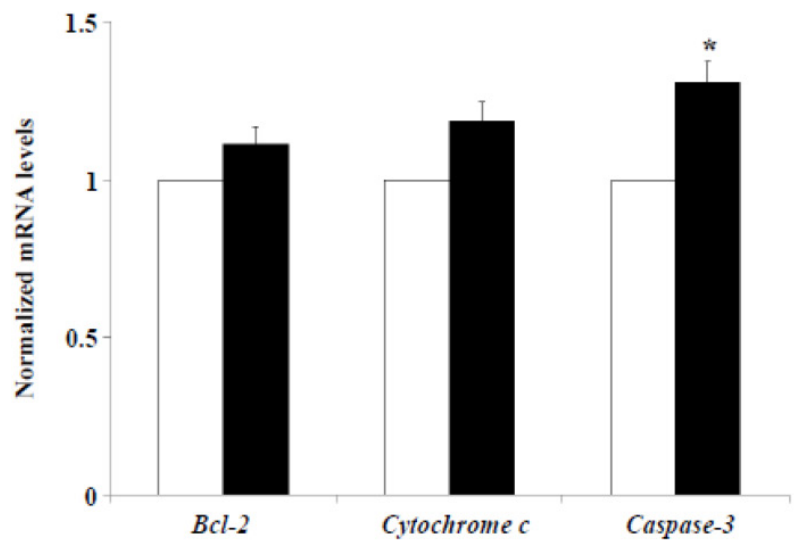

expressing scFv-M6-1B9 intrabody at day 10 compared with the irrelevant control cells, as illustrated in Figure 4B $(p<0.05)$. Thus, this result suggests that the down-regulation of EMMPRIN by the scFv-M6-1B9 intrabody significantly decreased CEA in both secreted and mRNA expression levels.

B

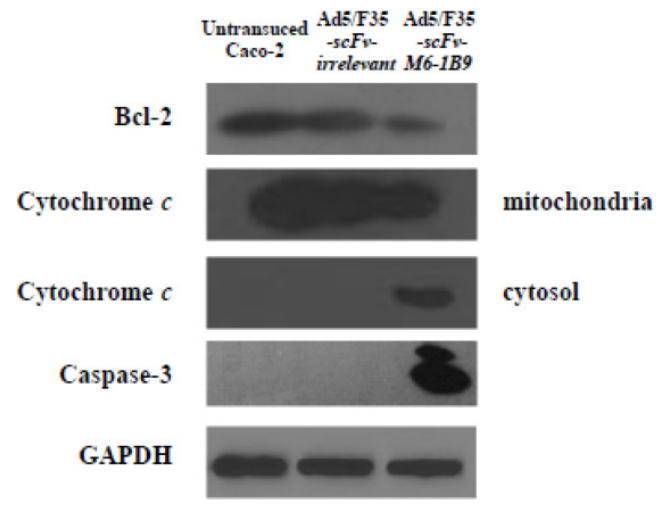

Figure 3. Activation of mitochondrial apoptosis signaling in EMMPRIN down-regulated Caco-2 cells. (A) Detection of Bcl-2, cytochrome $c$, and caspase-3 mRNA expression levels. Transduced Caco-2 cells were extracted for total RNA and reverse transcribed into cDNA. The expression of Bcl-2, cytochrome $c$, and caspase-3 mRNAs were analyzed using real-time RT-PCR analysis. GAPDH mRNA was used as the internal control. The normalized mRNA expression was analyzed using the comparative $C_{T}$ method. The white and black bars represent Ad5/F35-scFv-irrelevant and Ad5/F35-scFv-M6-IB9 transduced Caco-2 cells, respectively. The data are presented as mean \pm s.e.m. ${ }^{*} p<0.05$, as determined by one-way ANOVA. (B) The expression levels of Bcl-2, cytochrome $c$, and caspase-3 were determined by western blotting. The immunoblots were detected using rabbit anti-Bcl-2 mAb (or mouse anti-cytochrome $\mathrm{cmAb}$, and mouse anti-caspase-3 mAb), followed by peroxidase-conjugated goat anti-rabbit lgG pAb (or anti-mouse IgG pAb). GAPDH was used as the internal control.

A

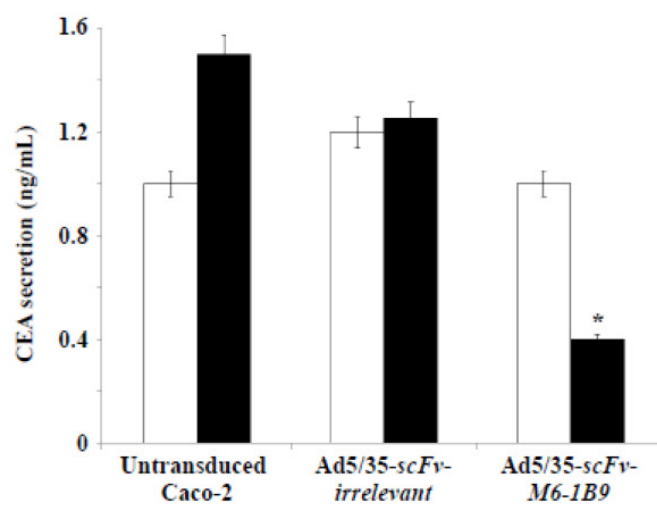

B

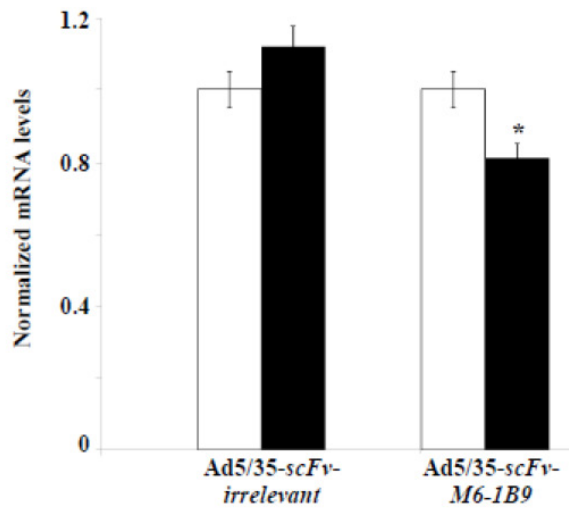

Figure 4. EMMPRIN down-regulation by scFv-M6-1B9 intrabody affecting decline of CEA level. Caco-2 cells transduced for 7 days and 10 days were collected as the supernatant, extracted cells for total RNA, and reverse transcribed into cDNA. (A) CEA secretions in the Caco-2 cell supernatant. The amount of CEA released into the medium was determined using an automated analyzer. (B) The expression of CEA mRNA was analyzed using real-time RT-PCR. GAPDH mRNA was used as the internal control. The normalized mRNA expression was analyzed using the comparative $C_{T}$ method. The white and black bars represent day 7 and day 10 of incubation, respectively. The data are presented as mean \pm s.e.m. $* p<0.05$, as determined by one-way ANOVA.

\section{Discussion}

EMMPRIN has been extensively studied and well established as playing a role in cancer progression [35]. Moreover, it has been characterized as a mediator of anti-apoptotic function and chemoresistance [36]. It has been reported that EMMPRIN can effectively protect apoptosis in oral squamous carcinoma cells and melanoma cell line A375 [37]. In addition, silencing EMMPRIN/CD147 mediated RNAi has been found to reduce X-chromosome linked inhibitor of apoptosis protein (XIAP) expression and increase chemosensitivity to 5-fluorouracil (5-FU) in human oral squamous carcinoma cells [38]. Therefore, 
the relevance of EMMPRIN as a target to induce apoptosis is shown to be significant as a valuable therapeutic in the case of cancers including colorectal cancer. These induce apoptosis directly, thus providing less opportunity for gained drug resistance, reduced mutagenesis, and toxicity [39].

In current study, we focused on functional activity of scFv-M6-1B9 intrabody counteract in situ EMMPRIN expression in Caco-2 cells. Cytoplasmic accumulation of the EMMPRIN molecule and significant decrease in the cell surface expression were found in $s c F v-M 6-1 B 9$ intrabody transduced Caco-2 cells. Hence, the particular advantage of the ER-targeted intrabody technology provides a simple and effective means of inhibiting the transport of integral membrane proteins to the cell surface. Based on the increasing number of in vitro and in vivo applications of ER-targeted intrabody, including in cancer [19, 40], infectious diseases [41], and autoimmune diseases [42], it is worth assessing its potential therapeutic efficacy.

This functional intrabody effects significant reduction in the EMMPRIN cell surface expression and perturbation as regards the proliferation property of the colorectal cancer cell, Caco-2. Furthermore, intracellular accumulation of MCT1 results in the increasing of intracellular lactate levels as a consequent effect of EMMPRIN down-regulation by scFv-M6-1B9 intrabody in Caco-2 cells [31]. We hypothesized that the disturbance of this molecule by decreased cell proliferation and intracellular accumulation of lactate might promote programmed cell death response in Caco-2 cells. Several studies have reported that the down-regulation of EMMPRIN/CD147 using siRNA technique increased apoptosis in head and neck squamous cell carcinoma (HNSCC) cell lines [43] and human umbilical vein endothelial cells (HUVECs) [9]. Our first observation is that down-regulation of EMMPRIN via scFv-M6-1B9 intrabody showed an increase in apoptotic cells, which was revealed by PI staining. This observation is based on the notion of PI, which is capable of binding DNA, which makes it probable to label fragmented DNA content of apoptotic cells [44]. During the progression of apoptosis, cells frequently exhibited phosphatidylserine in the outer layers of the plasma membrane, which has been translocated from the inner leaflet of plasma membrane [45]. Notably, this demonstrates the alteration of both early and late apoptotic cells, thus clearly indicating that Caco-2 cells expressing scFv-M6-1B9 intrabody had already started the apoptotic process with increased plasma membrane permeability to PI dye and Annexin V. The more pronounced effect of EMMPRIN down-regulation on apoptosis induction, measured by TUNEL assay, is presented in the DNA fragmented response, a morphological hallmark of apoptosis in the nucleus. In this study, we observed substantial increment of DNA fragmentation in the Caco-2 cells expressing scFv-M6-1B9 intrabody. These results are indicative of DNA damage, which could be one of the reasons for increased apoptotic cells and induced apoptosis processes observed in the down-regulation of EMMPRIN by the scFv-M6-1B9 intrabody.

The possible signaling of the molecules and the mechanisms involved in the apoptosis triggered by EMMPRIN down-regulation via scFv-M6-1B9 intrabody was also investigated. The particular importance of the effect of the intrabody against EMMPRIN is in its specific function inside the cell. We, therefore, focused on the mitochondrial (intrinsic) pathway in the EMMPRIN down-regulation induced apoptosis. It is well recognized that the mitochondrial pathway of apoptosis is regulated by the Bcl-2 family of proteins consisting of pro-apoptotic and anti-apoptotic proteins [46]. Bcl-2, an anti-apoptotic protein, is the central regulator of the mitochondrial outer membrane permeability and promotes the release of the cytochrome c[47]. In general, cytochrome $c$ is located in the mitochondrial intermembranous space and released into the cytosol when mitochondrial apoptosis is activated. It finally activates downstream caspase-3 to initiate apoptosis [48]. Our finding that Caco-2 cells expressing scFv-M6-1B9 intrabody demonstrate the reduction of Bcl-2 also provides evidence for the accumulation of cytochrome $c$ in the cytosolic compartment. In addition, the caspase-3 was found to significantly increase in both mRNA and protein expression levels. This observation suggests that the main mechanism of apoptosis response is through EMMPRIN down-regulation mediated scFv-M6-1B9 intrabody induction. The key function of this mechanism is the decrease in the level of the anti-apoptotic protein as a result of mitochondrial outer membrane permeabilization. This is followed by promotion of the cytochrome $c$ release from the mitochondria, which ultimately leads to downstream cleavage of executor caspase-3. These observations support our hypothesis that these molecules in the mitochondrial pathway are involved in the regulation of Caco-2 cell apoptotic regulation by way of EMMPRIN down-regulation.

Carcinoembryonic antigen (CEA) is the most widely used tumor marker worldwide, especially in colorectal cancer [49]. Recently, Peng et al. revealed through evaluation that the combination of CEA and Ki67 protein as a prognostic marker for colorectal cancer has better prognostic value than CEA by its own [50]. There have been reports that EMMPRIN cell surface expression also correlates with clinical prog- 
nosis of various human malignancies including breast cancer [51], pulmonary adenocarcinoma [52], and colorectal cancer [53, 54]. Interestingly, Caco-2 cells expressing scFv-M6-1B9 intrabody was observed to demonstrate statistically significant decrease in CEA expression at protein and mRNA levels. This reduction might have been caused by cell death or by the interference of secretory pathway in the down-regulation of EMMPRIN via the scFv-M6-1B9 intrabody. However, little is currently known about the underlying molecular mechanism. These results provide further insight into the role of EMMPRIN in CEA secretory pathway and its interaction. Moreover, developing these combinations might be used as prognostic markers in colorectal cancer.

It has been well established that EMMPRIN acts as a chaperone for monocarboxylate transporter 1 (MCT1) which is an accompaniment during the maturation in the ER and the translocation to the plasma membrane [55]. These interactions play an important role in lactate transport responsible for cellular $\mathrm{pH}$ regulation in relation to proliferation, transformation, and apoptosis [56-59]. The elevation of lactate and the further decrease in the intracellular $\mathrm{pH}\left(\mathrm{pH}_{\mathrm{i}}\right)$ are caused by the changes in the $\mathrm{H}^{+}$/lactate co-transporters which possess the ability to protect the cells from apoptosis by maintaining $\mathrm{pH}_{\mathrm{i}}$ [60]. It is convincingly evident that acidic $\mathrm{pH}_{\mathrm{i}}$ triggers the apoptosis hallmarks such as nuclear condensation, cytoplasmic vacuolization, and endonucleosome DNA degradation [61]. Our current study demonstrates that EMMPRIN and MCT1 translocation were defective in the Caco- 2 cells expressing the scFv-M6-1B9 intrabody. These cells effectively restrain the clearance of intracellular lactate and, ultimately, acidosis facilitates the occurrence of apoptosis. The possible mechanism of the acidic $\mathrm{pH}_{\mathrm{i}}$ potentially playing important roles in activating apoptosis is shown in three pathways: (i) by shifting the mitochondrial membrane potential $(\Delta \psi \mathrm{m})$ directly or promoting the binding of Bcl-2 to the Bcl-2 $19 \mathrm{kDa}$ interacting protein (BNIP3), which leads to increased mitochondrial membrane permeability [62]; (ii) by activating nuclear localized endonucleases involved in DNA fragmentation [63]; and (iii) by activating caspase-3, a pro-apoptotic protease that, in turn, inactivates proteins vital for normal cellular function [64]. Thus, the down-regulation of EMMPRIN by scFv-M6-1B9 intrabody induces apoptosis cell death in the colorectal cancer cell, Caco-2, and this is clearly associated with an elevated intracellular acidosis and eventually the consequence of the activation of apoptosis signaling molecules, as presented in Figure 5.
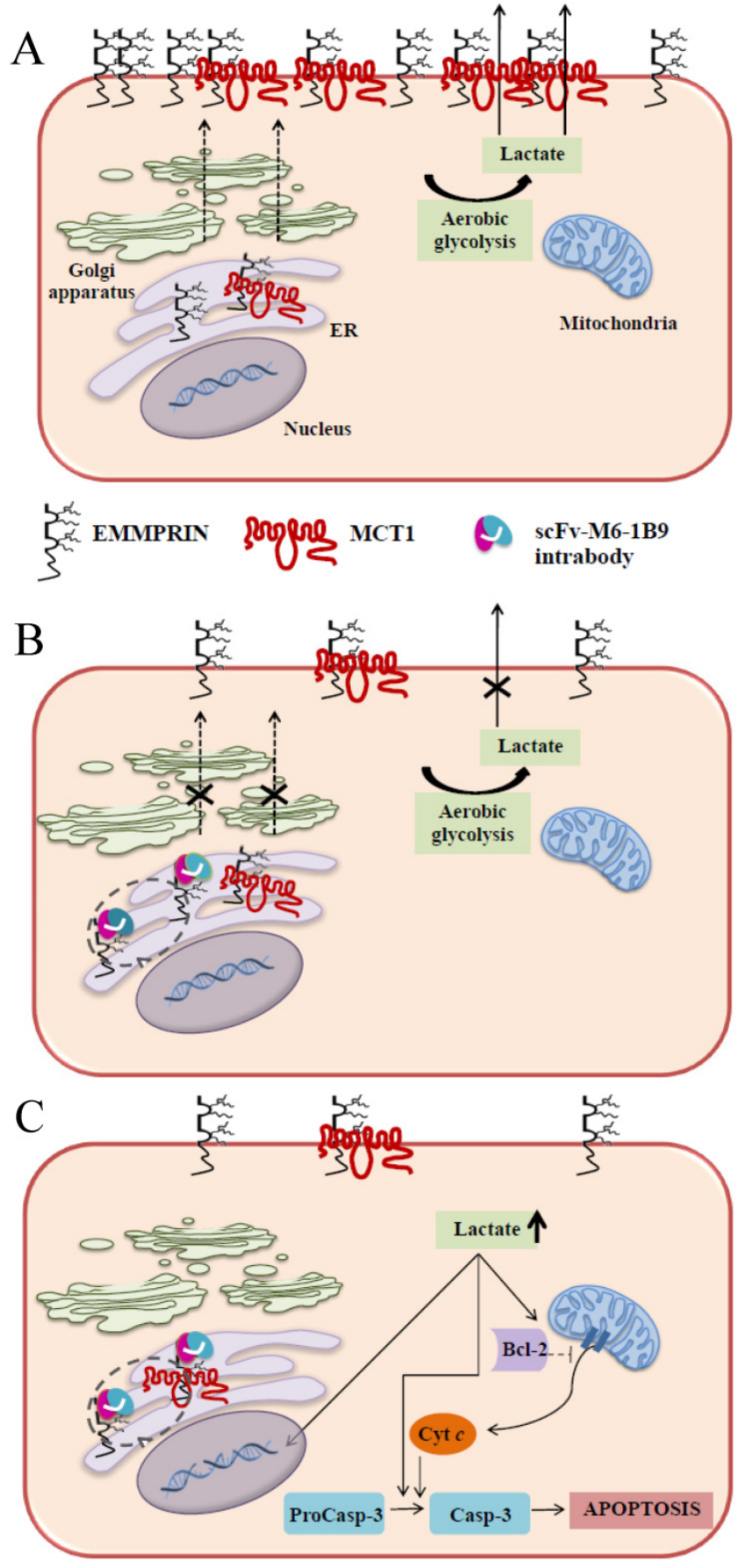

Figure 5. Schematic diagram of possible apoptotic mechanism underlying EMMPRIN down-regulation via scFv-M6-1B9 intrabody. (A) EMMPRIN facilitates proper expression of MCT1 at the cell surface. This association plays an important functional role in lactate transport. In cancer cells, an excess of lactic acid is produced by aerobic glycolysis, which must be rapidly exported across the plasma membrane to avoid a drop in $\mathrm{pH}_{\mathrm{i}}[57,65]$. (B) The scFv-M6-1B9 intrabody can specifically trap the EMMPRIN molecule within the ER, based on the ER retention signal. Suppression of EMMPRIN-MCTI complex by the down-regulation of EMMPRIN, which resulted in the inhibition of the lactate transporter. (C) Blocking of lactate transport leads to the accumulation of intracellular lactate inside the cell [31]. The acidic $\mathrm{pH}_{\mathrm{i}}$ can be stimulated by different pathways, which are as follows: (i) by altering the mitochondrial membrane potential $(\Delta \psi \mathrm{m})$ directly or by increasing the permeability of the outer mitochondrial membrane by promoting the binding of $\mathrm{Bcl}-2$ [62]; (ii) by activating nuclear localized endonucleases, a key enzyme that mediates regulated DNA fragmentation and chromatin condensation in response to apoptotic signals [63]; and (iii) by activating caspase-3, a pro-apoptotic cysteine protease involved in the activation cascade of caspases responsible for apoptosis execution [64]. 
In summary, the effective scFv-M6-1B9 intrabody is of paramount importance for down-regulating EMMPRIN cell surface expression on Caco-2 cells. Furthermore, this biologically active protein can potentially induce Caco-2 cell apoptosis with down-regulation of Bcl-2 via mitochondrial pathway stimulation. This is the first time that evidence of the association between EMMPRIN and CEA expression in colorectal cancer cells has been established. This could be considered to be supporting the notion of novel molecule involved in combined markers in the monitoring of colorectal cancer treatment. Thus, EMMPRIN down-regulation by scFv-M6-1B9 intrabody is evidently an attractive concept for targeted colorectal cancer gene therapy and colorectal cancer management.

\section{Acknowledgments}

This work was supported by the Discovery-based Development Grant, Research Chair Grant from the National Sciences and Technology Development Agency (NSTDA), the Thailand Research Fund (TRF), and the National Research University Project under the Thailand Office of the Commission on Higher Education, Thailand. We thank Prof. André Lieber from the Division of Medical Genetics, University of Washington, Seattle, USA, for providing Ad5/F35 vector; Assist. Prof. Dr. Yingmanee Tragoolpua from the Department of Biology, Faculty of Science, Chiang Mai University, Chiang Mai, Thailand, for providing apoptosis signaling antibodies; and Assist. Prof. Dr. Peeraya Munkhetvit from the Department of Occupational Therapy, Faculty of Associated Medical Sciences, Chiang Mai University, Chiang Mai, Thailand, for her advice on statistical analyses.

\section{Competing Interests}

The authors have declared that no competing interest exists.

\section{References}

1. Amaravadi RK, Lippincott-Schwartz J, Yin XM, et al. Principles and current strategies for targeting autophagy for cancer treatment. Clin Cancer Res. 2011; 17: 654-66.

2. Lee CS, Ryan EJ, and Doherty GA. Gastro-intestinal toxicity of chemotherapeutics in colorectal cancer: the role of inflammation. World J Gastroenterol. 2014; 20: 3751-61.

3. Kumar M, Nagpal R, Hemalatha R, et al. Targeted cancer therapies: the future of cancer treatment. Acta Biomed. 2012; 83: 220-33.

4. Ghobrial IM, Witzig TE, and Adjei AA. Targeting apoptosis pathways in cancer therapy. CA Cancer J Clin. 2005; 55: 178-94.

5. Ricci MS and Zong WX. Chemotherapeutic approaches for targeting cell death pathways. Oncologist. 2006; 11: 342-57.

6. Li X, Lu Y, Pan T, et al. Roles of autophagy in cetuximab-mediated cancer therapy against EGFR. Autophagy. 2010; 6: 1066-77.

7. Liang H, Zhan HJ, Wang BG, et al. Change in expression of apoptosis genes after hyperthermia, chemotherapy and radiotherapy in human colon cancer transplanted into nude mice. World J Gastroenterol. 2007; 13: 4365-71.

8. Jin A, Chen H, Wang C, et al. Elevated expression of CD147 in patients with endometriosis and its role in regulating apoptosis and migration of human endometrial cells. Fertil Steril. 2014; 101: 1681-7.
9. Xie W, Xie H, Liu F, et al. Propranolol induces apoptosis of human umbilical vein endothelial cells through downregulation of CD147. Br J Dermatol. 2013; 168: 739-48.

10. Chen H, Fok KL, Jiang X, et al. CD147 regulates apoptosis in mouse spermatocytes but not spermatogonia. Hum Reprod. 2012; 27: 1568-76.

11. Misra S, Ghatak S, Zoltan-Jones A, et al. Regulation of multidrug resistance in cancer cells by hyaluronan. J Biol Chem. 2003; 278: 25285-8.

12. Miyauchi T, Masuzawa $Y$, and Muramatsu T. The basigin group of the immunoglobulin superfamily: complete conservation of a segment in and around transmembrane domains of human and mouse basigin and chicken HT7 antigen. J Biochem. 1991; 110: 770-4.

13. Kasinrerk W, Fiebiger E, Stefanova I, et al. Human leukocyte activation antigen M6, a member of the Ig superfamily, is the species homologue of rat OX-47, mouse basigin, and chicken HT7 molecule. J Immunol. 1992; 149: 847-54.

14. Weidle UH, Scheuer W, Eggle D, et al. Cancer-related issues of CD147. Cancer Genomics Proteomics. 2010; 7: 157-69.

15. Yang JM, O'Neill $\mathrm{P}$, Jin $\mathrm{W}$, et al. Extracellular matrix metalloproteinase inducer (CD147) confers resistance of breast cancer cells to Anoikis through inhibition of Bim. J Biol Chem. 2006; 281: 9719-27.

16. Kontermann RE. Intrabodies as therapeutic agents. Methods. 2004; 34: 163-70.

17. Azemar M, Djahansouzi S, Jager E, et al. Regression of cutaneous tumor lesions in patients intratumorally injected with a recombinant single-chain antibody-toxin targeted to ErbB2/HER2. Breast Cancer Res Treat. 2003; 82: 155-64.

18. Yu F, Wang $\mathrm{Y}$, Xiao $\mathrm{Y}$, et al. RP215 single chain fragment variable and single domain recombinant antibodies induce cell cycle arrest at G0/G1 phase in breast cancer. Mol Immunol. 2014; 59: 100-9.

19. Popkov M, Jendreyko N, McGavern DB, et al. Targeting tumor angiogenesis with adenovirus-delivered anti-Tie-2 intrabody. Cancer Res. 2005; 65: 972-81.

20. Nejatollahi F, Abdi S, and Asgharpour M. Antiproliferative and apoptotic effects of a specific antiprostate stem cell single chain antibody on human prostate cancer cells. J Oncol. 2013; 2013: 1-8.

21. Sagawa M, Shimizu T, Fukushima N, et al. A new disulfide-linked dimer of a single-chain antibody fragment against human CD47 induces apoptosis in lymphoid malignant cells via the hypoxia inducible factor-1alpha pathway. Cancer Sci. 2011; 102: 1208-15.

22. Intasai N, Tragoolpua K, Pingmuang $\mathrm{P}$, et al. Potent inhibition of OKT3-induced T cell proliferation and suppression of CD147 cell surface expression in HeLa cells by scFv-M6-1B9. Immunobiology. 2009; 214: 410-21.

23. Tragoolpua K, Intasai N, Kasinrerk W, et al. Generation of functional scFv intrabody to abate the expression of CD147 surface molecule of 293A cells. BMC Biotechnol. 2008; 8: 1-13.

24. Romano G, Michell P, Pacilio C, et al. Latest developments in gene transfer technology: achievements, perspectives, and controversies over therapeutic applications. Stem Cells. 2000; 18: 19-39.

25. Bouard D, Alazard-Dany D, and Cosset FL. Viral vectors: from virology to transgene expression. Br J Pharmacol. 2009; 157: 153-65.

26. Connolly JB. Lentiviruses in gene therapy clinical research. Gene Ther. 2002; 9: 1730-4.

27. Rux JJ and Burnett RM. Adenovirus structure. Hum Gene Ther. 2004; 15: 1167-76.

28. Rein DT, Breidenbach $\mathrm{M}, \mathrm{Wu} \mathrm{H}$, et al. Gene transfer to cervical cancer with fiber-modified adenoviruses. Int J Cancer. 2004; 111: 698-704.

29. Yotnda P, Onishi H, Heslop HE, et al. Efficient infection of primitive hematopoietic stem cells by modified adenovirus. Gene Ther. 2001; 8: 930-7.

30. Nilsson M, Ljungberg J, Richter J, et al. Development of an adenoviral vector system with adenovirus serotype 35 tropism; efficient transient gene transfer into primary malignant hematopoietic cells. J Gene Med. 2004; 6: 631-41.

31. Sangboonruang $\mathrm{S}$, Thammasit $\mathrm{P}$, Intasai $\mathrm{N}$, et al. EMMPRIN reduction via scFv-M6-1B9 intrabody affects alpha3beta1-integrin and MCT1 functions and results in suppression of progressive phenotype in the colorectal cancer cell line Caco-2. Cancer Gene Ther. 2014; 21: 246-55.

32. Livak KJ and Schmittgen TD. Analysis of relative gene expression data using real-time quantitative PCR and the 2(-Delta Delta C(T)) Method. Methods. 2001; 25: 402-8.

33. Pakdel A, Naghibalhossaini F, Mokarram P, et al. Regulation of carcinoembryonic antigen release from colorectal cancer cells. Mol Biol Rep. 2012; 39: 3695-704.

34. Jonas S, Windeatt S, A OB, et al. Allen-Mersh. Identification of carcinoembryonic antigen-producing cells circulating in the blood of patients with colorectal carcinoma by reverse transcriptase polymerase chain reaction. Gut. 1996; 39: 717-21.

35. Kanekura T, Chen X, and Kanzaki T. Basigin (CD147) is expressed on melanoma cells and induces tumor cell invasion by stimulating production of matrix metalloproteinases by fibroblasts. Int J Cancer. 2002; 99: 520-8.

36. Tang J, Guo YS, Zhang Y, et al. CD147 induces UPR to inhibit apoptosis and chemosensitivity by increasing the transcription of Bip in hepatocellular carcinoma. Cell Death Differ. 2012; 19: 1779-90.

37. Sommers Smith SK and Smith DM. Beta blockade induces apoptosis in cultured capillary endothelial cells. In Vitro Cell Dev Biol Anim. 2002; 38: 298-304.

38. Kuang YH, Chen X, Su J, et al. RNA interference targeting the CD147 induces apoptosis of multi-drug resistant cancer cells related to XIAP depletion. Cancer Lett. 2009; 276: 189-95.

39. Lowe SW and Lin AW. Apoptosis in cancer. Carcinogenesis. 2000; 21: 485-95. 
40. Figini M, Ferri R, Mezzanzanica D, et al. Reversion of transformed phenotype in ovarian cancer cells by intracellular expression of anti folate receptor antibodies. Gene Ther. 2003; 10: 1018-25.

41. Walsh R, Nuttall S, Revill P, et al. Targeting the hepatitis B virus precore antigen with a novel IgNAR single variable domain intrabody. Virology. 2011; 411: 132-41.

42. Reimer E, Somplatzki S, Zegenhagen D, et al. Molecular cloning and characterization of a novel anti-TLR9 intrabody. Cell Mol Biol Lett. 2013; 18: 433-46.

43. Huang Z, Wang L, Wang Y, et al. Overexpression of CD147 contributes to the chemoresistance of head and neck squamous cell carcinoma cells. J Oral Pathol Med. 2013; 42: 541-6.

44. Crompton T, Peitsch MC, MacDonald HR, et al. Propidium iodide staining correlates with the extent of DNA degradation in isolated nuclei. Biochem Biophys Res Commun. 1992; 183: 532-7.

45. Hengartner MO. The biochemistry of apoptosis. Nature. 2000; 407: 770-6

46. Merry DE and Korsmeyer SJ. Bcl-2 gene family in the nervous system. Annu Rev Neurosci. 1997; 20: 245-67.

47. Reed JC. Proapoptotic multidomain Bcl-2/Bax-family proteins: mechanisms, physiological roles, and therapeutic opportunities. Cell Death Differ. 2006; 13: 1378-86.

48. Green D and Kroemer G. The central executioners of apoptosis: caspases or mitochondria? Trends Cell Biol. 1998; 8: 267-71.

49. Duffy MJ. Carcinoembryonic antigen as a marker for colorectal cancer: is it clinically useful? Clin Chem. 2001; 47: 624-30.

50. Peng Y, Wang L, and Gu J. Elevated preoperative carcinoembryonic antigen (CEA) and Ki67 is predictor of decreased survival in IIA stage colon cancer. World J Surg. 2013; 37: 208-13.

51. Stenzinger A, Wittschieber D, Winterfeld M, et al. High extracellular matrix metalloproteinase inducer/CD147 expression is strongly and independently associated with poor prognosis in colorectal cancer. Hum Pathol. 2012; 43: 1471-81.

52. Zhong X, Li M, Nie B, et al. Overexpressions of RACK1 and CD147 associated with poor prognosis in stage T1 pulmonary adenocarcinoma. Ann Surg Oncol. 2013; 20: 1044-52.

53. Zhu S, Chu D, Zhang Y, et al. EMMPRIN/CD147 expression is associated with disease-free survival of patients with colorectal cancer. Med Oncol. 2013; 30: 369.
54. Boye K, Nesland JM, Sandstad B, et al. EMMPRIN is associated with S100A4 and predicts patient outcome in colorectal cancer. Br J Cancer. 2012; 107: 667-74.

55. Deora AA, Philp N, Hu J, et al. Mechanisms regulating tissue-specific polarity of monocarboxylate transporters and their chaperone CD147 in kidney and retinal epithelia. Proc Natl Acad Sci U S A. 2005; 102: 16245-50.

56. Kirk P, Wilson MC, Heddle C, et al. CD147 is tightly associated with lactate transporters MCT1 and MCT4 and facilitates their cell surface expression. EMBO J. 2000; 19: 3896-904

57. Halestrap AP and Price NT. The proton-linked monocarboxylate transporter (MCT) family: structure, function and regulation. Biochem J. 1999; 343: 281-99.

58. Ferreira LM. Cancer metabolism: the Warburg effect today. Exp Mol Pathol. 2010; 89: 372-80.

59. Zhao Y, Butler EB, and Tan M. Targeting cellular metabolism to improve cancer therapeutics. Cell Death Dis. 2013; 4: 1-10.

60. Shrode LD, Tapper H, and Grinstein S. Role of intracellular $\mathrm{pH}$ in proliferation, transformation, and apoptosis. J Bioenerg Biomembr. 1997; 29: 393-9.

61. Park HJ, Makepeace CM, Lyons JC, et al. Effect of intracellular acidity and ionomycin on apoptosis in HL-60 cells. Eur J Cancer. 1996; 32: 540-6.

62. Matsuyama S, Llopis J, Deveraux QL, et al. Changes in intramitochondrial and cytosolic $\mathrm{pH}$ : early events that modulate caspase activation during apoptosis. Nat Cell Biol. 2000; 2: 318-25.

63. Barry MA and Eastman A. Endonuclease activation during apoptosis: the role of cytosolic Ca2+ and pH. Biochem Biophys Res Commun. 1992; 186: 782-9.

64. Park HJ, Lyons JC, Ohtsubo T, et al. Acidic environment causes apoptosis by increasing caspase activity. Br J Cancer. 1999; 80: 1892-7.

65. Poole RC and Halestrap AP. Transport of lactate and other monocarboxylates across mammalian plasma membranes. Am J Physiol. 1993; 264: 761-82.

66. Sun KW, Ma YY, Guan TP, et al. Oridonin induces apoptosis in gastric cancer through Apaf-1, cytochrome $c$ and caspase-3 signaling pathway. World J Gastroenterol. 2012; 18: 7166-74.

67. Shang LH, Li CM, Yang ZY, et al. Luffa echinata Roxb. induces human colon cancer cell (HT-29) death by triggering the mitochondrial apoptosis pathway. Molecules. 2012; 17: 5780-94.

68. Janssens N, Janicot M, Perera $\mathrm{T}$, et al. Housekeeping genes as internal standards in cancer research. Mol Diagn. 2004; 8: 107-13. 